\title{
What State Health Officials Wish They Had Known and How They Learned Best
}

Baker, Edward L. MD, MPH; Castrucci, Brian C. MA; Moffatt, Sharon MS, BSN, RN; Babich,

Suzanne M. DrPH, MS; Boedigheimer, Steven F. MBA; Hancock, Suzanne E. MPH; Tilson, Hugh MD, MPH; Yeagar, Valerie A. DrPH; Halverson, Paul DrPH, FACHE

Author Information

Indiana University Richard M. Fairbanks School of Public Health, Indianapolis, Indiana (Drs Baker, Babich, Halverson, Tilson, and Yeagar, Ms Hancock, and Mr

Boedigheimer); de Beaumont Foundation, Bethesda, Maryland (Mr Castrucci); and Association of State and Territorial Health Officials, Arlington, Virginia (Ms Moffatt).

Correspondence: Edward L. Baker, MD, MPH, 25 Vassal Lane, Cambridge, MA 02138 (edwardlbaker@gmail.com).

The authors declare no conflicts of interest.

In a prior Management Moment column, ${ }^{1}$ which introduced our study of state and territorial health official success and critical success factors, we summarized a few important themes derived from informal group interviews with state health officials (SHOs) and senior deputies. They identified 2 aspects of success: (1) specific policy or program achievements, and (2) team building within the state health agency. A range of critical success factors including individual attributes and skills and on-the-job behaviors was perceived to contribute to these successes. Organizational structure and culture also were perceived to impact the ability of SHOs to achieve success.

This is the author's manuscript of the article published in final edited form as:

Baker, E. L., Castrucci, B. C., Moffatt, S., Babich, S. M., Boedigheimer, S. F., Hancock, S. E., ... Halverson, P. (2018). What State Health Officials Wish They Had Known and How They Learned Best. Journal of Public Health Management and Practice, 24(1), 85-86. https://doi.org/10.1097/PHH.0000000000000695 
Most SHOs function as the chief executive officers (CEOs) of their health departments. However, unlike most CEOs, many SHOs come to the position not having had extensive managerial experience and, likewise, lack experience in a governmental setting, in public health, or both. This presents unique challenges for new SHOs. Given the importance of this role in achieving effective departmental operation as well as the optimal health of the public, we have been conducting interviews to better understand what SHOs know and don't know before taking the job and how they learned best once in the position.

In this column, we begin the process of sharing insights from our ongoing research project on SHO success. In doing so, we explore perceptions of current and past SHOs whom we formally interviewed about those things SHOs wish they had known before taking the position and also steps they took during their first several months on the job to enhance their effectiveness and impact.

Although our research relates specifically to the experiences of SHOs, some of our findings are relevant to other public health leadership positions. In that regard, a prior Management Moment column relating to actions needed during the first 6 months on the job may be of interest to readers. ${ }^{2}$

\section{What Current SHOs Wish They Had Known}

When asked what they wish they had known before taking the job, SHOs appeared to answer differently based on their prior state government experience. Answers to this question varied depending upon whether the SHO was selected from outside government or from inside state government. Nevertheless, several consistent themes emerged but with greater emphasis provided among those who were new to state government. They included the following: 
- Better understanding of the political process: Many SHOs indicated a need to better understand how to relate to the governor's office. This included how best to understand the governor's priorities and also how best to relate to the governor's senior staff. Furthermore, SHOs indicated a need to better understand, relate to, and influence the legislative process including processes for working with state legislators and their senior staff.

- Better understanding of how state government works: SHOs highlighted a strong need to better understand governmental budgeting and finance including how best to defend the agency's budget. Managing change within government was another noted challenge including specific business process management techniques, overcoming silo issues, and changing organizational culture. SHOs noted a need to better master workforce development strategies including leadership team building, understanding governmental personnel systems, and dealing with problem employees. Finally, a need to enhance general management skills was noted such as how to have better meetings and better communication skills, including risk communication.

- Better understanding of partnership development approaches: SHOs indicated an interest in knowing more about cross-sectoral partnership development approaches, along with ways to work more effectively with other agencies of state government (eg, Medicaid, transportation, education, environment). SHOs particularly expressed a need to know more about relationships with the federal government including grants and other policy issues. One identified source of enhancing understanding of these and other areas was learning from SHOs in other states.

\section{How SHOs Learned Best}


Since many SHOs entered their position without some of the understandings noted earlier, the first few months on the job are particularly crucial. ${ }^{3,4}$ Our research is exploring both those processes that were helpful to the SHO in enhancing his or her knowledge and understanding and those procedures that enabled others to better understand the background and priorities of the SHO.

- Processes helpful to enhance SHO knowledge: SHOs found that formal briefings along with the practice of "management by walking around" to be of greatest value as they learned about SHO roles and responsibilities. Of great value was access to a person responsible for orientation to the job (eg, senior deputy) along with meetings with constituency groups.

- Processes that helped others learn about the SHO: Meetings with key senior state health agency staff individually or in a group were felt to help SHOs introduce themselves and share priorities. Visiting agency programs and meeting staff were also important. Many SHOs stated that they mostly listened and limited information delivery during the first several weeks. In some instances, a formal videotaped introduction or "all hands" meeting by the SHO was an efficient tool to help agency staff learn about the SHO.

\section{Conclusion}

To maximize the impact of SHOs in their roles, it is important to increase their likelihood of success as much as possible. These preliminary findings identify 3 areas that, if addressed early, could help improve SHO effectiveness. Especially unique to the SHO role is the entry into the political process. This is likely a commonality that nearly all first-time SHOs will share and 
should therefore be addressed early in the new SHO's tenure. However, it is likely that many SHOs are left to learn political skills "on the job,” placing their success in jeopardy.

Our ongoing research on SHO success and critical success factors has begun to identify which areas SHOs wish they understood better before taking on the task of leading the health agency and also a few processes that were useful to new SHOs as they took office. As our research study evolves, we will continue to share other insights into best practices and guiding principles for this key leadership position.

\section{References}

1. Halverson P, Castrucci BC, Moffatt S, Hancock SE, Boedigheimer SF, Baker EL. State health officials—defining success and identifying critical success factors. J Public Health Manag Pract. 2017;23(2):192-194.

2. Ruiz R, Baker EL. The first 6 months: transitioning into a new position. J Public Health Manag Pract. 2013;19(2):187-189.

3. Atchison CG, Beitsch LM, Benjamin GC, et al First Days: A guide for State and Territorial Health Officials. Arlington, VA: Association of State and Territorial Health Officials; 2009.

4. Daly PH, Watkins M. The First 90 Days in Government. Boston, MA: Harvard Business Review Press; 2006. 